\title{
The impact of clinical factor on dosimetry in radioembolisation
}

\author{
M. Maccauro $^{1} \cdot$ R. Salem ${ }^{2}$
}

Received: 1 April 2016/Accepted: 27 May 2016/Published online: 21 June 2016

(C) Italian Association of Nuclear Medicine and Molecular Imaging 2016

Primary liver cancer represents the fifth most common cancer in the world and the third most common cause of cancer mortality. Furthermore liver is the most site of metastases and up to $25 \%$ of patients with oncological malignancies develop hepatic lesions, especially from colorectal, pancreatic and breast cancer. Only a minority of patients, less than $10 \%$, with hepatocellular carcinoma (HCC) or liver metastases are candidates for radical treatment due to stage of presentation, hepatic disfunction or co-morbidities. Local treatment, as radiofrequency ablation (RFA), transarterial chemoembolization (TACE) and transarterial radioembolization (TARE) are alternative treatment in patients with unresectable disease.

Radioembolisation is a well-known and described therapeutic procedure based on intra-arterial injection of micro-spheres of glass or resin loaded with the radioactive isotope Yttrium-90. This is a pure beta emitter with the maximal beta energy among the nuclide for therapy of $2280 \mathrm{keV}$, corresponding to a maximum range of $11 \mathrm{~mm}$ in soft tissues and a physical half-life of $64.2 \mathrm{~h}$. TARE is an emerging and promising approach of nuclear medicine therapy. It takes advantage of the predominant arterial vascularisation typical of focal liver lesions and of the small size of the spheres (around 25 microns). Indeed micro-particles carried by the arterial flow remain trapped in the arterioles and capillaries of the tumour and release

M. Maccauro

marco.maccauro@istitutotumori.mi.it

1 Nuclear Medicine Division, Foundation IRCCS Istituto Nazionale Tumori, Milan, Italy

2 Department of Radiology, Northwestern University, Chicago, USA their energy there. The final result is a highly targeted and selective intra-arterial radiotherapy.

The role and the clinical impact of TARE is under investigation in phase III randomized international multicenter trials [NCT0095930; NCT01482442; NCT01135056; NCTO 1126645; NCT01887717; NCT01556490; NCT00867750] in order to define its proper allocation as standard of care.

To accomplish this selective and effective therapy, it is mandatory to simulate the treatment through the study of the biodistribution of particles similar to the therapeutic microspheres. ${ }^{99 \mathrm{~m}}$ Tc- macroaggregated albumin (MAA) are administered for the simulation, and planar scintigraphy and abdominal SPECT/CT scan are performed. This diagnostic procedure helps to exclude the presence of extrahepatic gastrointestinal shunts (absolute contraindication to the treatment), to quantify the lung shunting percentage, and permits pre-treatment dose calculation (3D treatment planning).

The success of this multidisciplinary therapy is based on several elements. It primarily depends on the capability to perform selective microsphere injections into tumors, translating to improved perfusion and higher tumor dose. The selective injection also allows a reduction of liver toxicity. The ideal situation is when the treatment covers the entire arterial circulation of the cancer, so as to leave no part excluded, preserving as much of the cancer-free parenchyma as possible. For this reason, it is of fundamental importance to identify the best point for the infusion of micro-spheres during the angiographic workup. To maximize the tumor dose reducing the liver impairment risk, it is better to treat as selectively as possible i.e., in a segmental, multi-segmental or lobar manner. This allows the regeneration of the untreated portion to compensate for the radiated treated lobe. 
The other novelty of TARE is the attention paid by many studies to its dosimetric aspects. Dosimetry can be easily calculated before the treatment, using the ${ }^{99 m} \mathrm{Tc}$ MAA SPECT/CT images of the simulation session, and after the therapeutic administration as confirmation, using either ${ }^{90} \mathrm{Y}$ bremsstrahlung SPECT or more quantitatively accurate ${ }^{90} \mathrm{Y}$ positron emission tomography (PET) images. These two dosimetric approaches have different aims and philosophy. The goal of pre-therapeutic dosimetry is to choose the optimal activity for each patient and deliver a tumoricidal absorbed dose to tumors while sparing the healthy liver tissue. Individualization is a leitmotif of modern medicine. Individual optimization of the treatment, i.e. pursuing the best efficacy and lowest toxicity profile, requires that the tumor absorbed dose (TD) and that absorbed by healthy injected liver tissue (HILD) should be estimated prior to administering the therapy.

Several dosimetric approaches have been described for glass and resin microspheres in $\mathrm{HCC}$ and liver metastases (A-B-C-D). Many clinical factors have to be evaluated since they may have an impact on pre-therapeutic MAA SPECT/CT dosimetry:

- Biological aspect (e.g., primary tumor or metastases). In HCC, baseline Child-Pugh results in different risks of adverse events. Patients with Child-Pugh class A show lower complication rate than Child B7. In latter case the ${ }^{99 m}$ Tc-MAA perfusion should ideally be concentrated in the tumour. Otherwise it is mandatory to reduce the activity to reduce the absorbed dose to the non tumoral tissue. Another risk factor for toxicity beyond the parenchyma absorbed dose was ascertained by Garin et al. [1]: infiltrative tumours with portal vein thrombosis (PVT) not perfused by MAA. Caution should therefore be applied in patients with untargeted PVT or grade IIIb or IV (complete obstruction). For metastases, the clinical situation differs. The randomized and multicenter study SIRFLOX (http://jco. ascopubs.org/cgi/doi/10.1200/JCO.2015.66.1181) has demonstrated that the combination with TARE in patients who received previous or concomitant chemotherapy may increase toxicity. In these patients the pre-therapeutic dosimetry can help to calculate the optimal dose.

- Hepatic reserve The importance of the fractional volume of irradiated versus non-irradiated liver is well known from external beam radiotherapy. In TARE, liver toxicities are observed solely in cases involving whole-liver injection, but rarely in those treated with lobar injection. Therefore, the best approach is the hyper-selective (segmental) injection using high doses resulting in low toxicity profile, as was first described by Riaz et al. [2].
- Mismatch between ${ }^{99 m} T c-M A A$ and ${ }^{90} Y$ microspheres this is caused by intentional changes in catheter positioning, attempting to perform more selective injections, or by inadvertent mispositioning, by spasm occurrence in one of the two angiographies (especially after coiling, prolonged procedure or use of rigid catheter) recently identified as possible explanation of mismatch by Garin et al. [3] or by differences in size or number between the simulation and therapeutic particles. This situation impacts predictive power of the dosimetric calculation. This mismatch has not prevented the finding of a dose-effect correlation, with all methods employed. Therefore, a minimally invasive as possible angiographic procedure and the verification by ${ }^{90} \mathrm{Y}$ PET or bremsstrahlung are highly recommended.

- Lung shunt The severity of radiomicrosphere lung injury depends on the fractional amount of hepatopulmonary shunt, on the lung radiation absorbed dose, on and pulmonary biodistribution. The lung dose-toxicity relationship is currently under investigation, because the size difference between MAA and microsphere, the difference of lung parenchyma standard mass $(1 \mathrm{~kg})$ with respect to the individual effective mass, and the lung dosimetry method (planar scintigraphy versus SPECT/ CT methodology) with other kind of microspheres play a major role. One other aspect to keep in mind is that the $1 \mathrm{~kg}$ of lung mass is modeled, and in fact may be refined in patients with very large or small lung volumes.

- Aim of treatment the potentially curative or palliative nature of treatment has to be decided before choosing the therapeutic activity. By experience from Milan group [4], favourable cases (high predicted tumour dose close to $500 \mathrm{~Gy}$, small tumours, good perfusion of PVT, no lung shunt) can be administered with curative intent close to the $75 \mathrm{~Gy}$ whole parenchyma limit, accepting a $15 \%$ risk of liver decompensation. In unfavourable cases (poor tumour perfusion, large tumours, tumours with hypoperfused regions, poor PVT perfusion, PVT IIIb or IV), dose reduction appears safer to reduce the administered activity with respect to the 75 Gy limit, reducing the LD risk with the goal of a palliative treatment.

In conclusion, the ${ }^{99 \mathrm{~m}} \mathrm{Tc}-\mathrm{MAA}$ SPECT/CT-based dosimetry is important in TARE, since it allows the individual optimization of treatment from the patient radiation protection point of view, as required by the current and future legislation (Council Directive 2013/59, art. 56 "optimization"). [http://eur-lex.europa.eu/legal-content/ EN/TXT/PDF/?uri=CELEX:32013L0059\&from=EN]. However, dosimetric evaluations should be accompanied 
by the above mentioned clinical considerations to perform patient tailored treatment.

\section{Compliance with ethical standards}

Conflict of interest Marco Maccauro and Riad Salem are consultants for BTG International.

Ethical approval This article does not contain any studies with human participants or animals performed by any of the authors.

\section{References}

1. Garin E, Lenoir L, Edeline J, Laffont S, Mesbah H, Porée P, Sulpice L, Boudjema K, Mesbah M, Guillygomarc'h A, Quehen E, Pracht M, Raoul JL, Clement B, Rolland Y, Boucher E (2013) Boosted selective internal radiation therapy with 90Y-loaded glass microspheres (B-SIRT) for hepatocellular carcinoma patients: a new personalized promising concept. Eur $\mathrm{J}$ Nucl Med Mol Imaging 40:1057-1068
2. Riaz A, Gates VL, Atassi B, Lewandowski RJ, Mulcahy MF, Ryu RK, Sato KT, Baker T, Kulik L, Gupta R, Abecassis M, Benson AB 3rd, Omary R, Millender L, Kennedy A, Salem R (2011) Radiation segmentectomy: a novel approach to increase safety and efficacy of radioembolization. Int $\mathbf{J}$ Radiat Oncol Biol Phys 79(1):163-171. doi:10.1016/j.ijrobp.2009.10.062 (epub 2010 Apr 24)

3. Garin E, Rolland Y, Laffont S, Edeline J (2016) Clinical impact of (99m)Tc-MAA SPECT/CT-based dosimetry in the radioembolization of liver malignancies with (90)Y-loaded microspheres. Eur J Nucl Med Mol Imaging 43(3):559-575. doi:10.1007/s00259-0153157-8 (epub 2015 Sep 4)

4. Chiesa C, Mira M, Maccauro M, Spreafico C, Romito R, Morosi C, Camerini T, Carrara M, Pellizzari S, Negri A, Aliberti G, Sposito C, Bhoori S, Facciorusso A, Civelli E, Lanocita R, Padovano B, Migliorisi M, De Nile MC, Seregni E, Marchianò A, Crippa F, Mazzaferro V (2015) Radioembolization of hepatocarcinoma with (90)Y glass microspheres: development of an individualized treatment planning strategy based on dosimetry and radiobiology. Eur J Nucl Med Mol Imaging 42(11):1718-1738. doi:10.1007/ s00259-015-3068-8 (epub 2015 Jun 27) 